\title{
Enhanced characterization of niobium surface topography
}

\author{
Chen Xu, ${ }^{1,2}$ Hui Tian, ${ }^{1}$ Charles E. Reece, ${ }^{1}$ and Michael J. Kelley ${ }^{1,2}$ \\ ${ }^{1}$ Thomas Jefferson National Accelerator Facility, Newport News, Virginia 23606, USA \\ ${ }^{2}$ Department of Applied Science, College of William \& Mary, Williamsburg, Virginia 23187-8795, USA
}

(Received 6 May 2011; published 6 December 2011)

Surface topography characterization is a continuing issue for the superconducting radio frequency (SRF) particle accelerator community. Efforts are under way to both improve surface topography and its characterization and analysis using various techniques. In measurement of topography, power spectral density (PSD) is a promising method to quantify typical surface parameters and develop scale-specific interpretations. PSD can also be used to indicate how the process modifies topography at different scales. However, generating an accurate and meaningful topographic PSD of an SRF surface requires careful analysis and optimization. In this report, niobium surfaces with different process histories are sampled with atomic force microscopy and stylus profilometry and analyzed to trace topography evolution at different scales. An optimized PSD analysis protocol to serve SRF needs is presented.

PACS numbers: $82.45 . \mathrm{Rr}, 82.80 . \mathrm{Fk}, 81.65 . \mathrm{Ps}$

\section{BACKGROUND AND MOTIVATION}

Particle accelerators continue to grow in importance as tools for scientific research, with applications from the most fundamental physics to user light sources. In many applications, superconducting radiofrequency (SRF) accelerator technology delivers improved performance over traditional technology such that SRF's role continues to expand. The ever-scarce resources for new facilities could go further if their cost could be reduced on a relative basis by increasing SRF performance. The performance of such accelerating structures is usually described by plotting the cavity quality factor $Q_{o}$ versus the accelerating gradient $E_{\text {acc }}$ [1]. In terms of impact, $Q_{o}$ is the ratio of the energy stored in the cavity to the energy dissipated in its walls, energy that must be removed by the cryosystem. Gradient determines how much accelerator length is needed to achieve the chosen exit beam energy. Gradient is the performance challenge for a high energy pulsed accelerator such as the proposed International Linear Collider. For continuous wave accelerators of both large and small scale, minimization of dissipated power (maximizing $Q_{o}$ ), or more generally minimizing cryogenic costs, is vital. Two types of performance deficits observed in the push to everhigher gradients are: (i) a decrease in $Q_{o}$ with increasing gradient- " $Q$ drop," when a certain gradient is reached; (ii) a lower $Q_{o}$ than expected at all gradients.

Surface topography has long been viewed as a major contributor via magnetic field enhancement (e.g., at grain boundary edges) [2]. Even when this enhancement is not sufficient to induce a full quench of the resonance, it may

Published by the American Physical Society under the terms of the Creative Commons Attribution 3.0 License. Further distribution of this work must maintain attribution to the author(s) and the published article's title, journal citation, and DOI. change the local loss. Though the resulting normalconducting volume may be too small to trigger a general quench, the cumulative effect of many such small defects turning on with increasing gradient would be manifest as reducing $Q_{o}$ [3] at a higher gradient. Recent studies [4] emphasize the importance of intrusions (pits, grain boundary crevices) as well as protrusions. Some of the computational modeling results are surprising. For example a "sharp-edged" (few-micron radius) shallow (depth/diameter $<0.5$ ) disk-shaped pit would have a field enhancement factor between 1.5 and 2 [2,5,6]. Chemically produced etching features on niobium with edge radius of curvature as low as $50 \mathrm{~nm}$ have been reported [5]. A recent theoretical analysis [6] suggests that thermal feedback may make even apparently modest defects more pernicious than once thought. A small amount of power deposited at the defect raises the local temperature slightly, increasing the population of normal-conducting electrons. The local surface resistance increases, which further increases power deposition until either heat transport out of the absorbing region limits further temperature increase or a quench occurs. Efforts to explicitly model the effect of topography continue [7]. Nonetheless, a clear consensus exists in the SRF community that smoother is better and that a more sophisticated analysis is called for.

The topography present on the interior surface of SRF cavities arises from the action of the preparation steps on the typical fine-grained $(\sim 50 \mu \mathrm{m}$ grain size) niobium sheet material from which they are made. As described at length elsewhere [8], the sheet stock is cut, shaped, and welded, then polished mechanically and chemically etched. Other forms of mechanical polishing are being replaced by centrifugal barrel polishing, wherein a specially designed abrasive medium is placed within the cavity and then tumbled to obtain a uniform surface condition. Cavity etching has been accomplished by buffered 
chemical polish (BCP), consisting of flowing a 1:1:2 mixture of hydrofluoric, nitric, and phosphoric acids through the cavity at $10^{\circ} \mathrm{C}$, removing a few microns per minute up to a total of about $100 \mu \mathrm{m}$. Seeking greater final
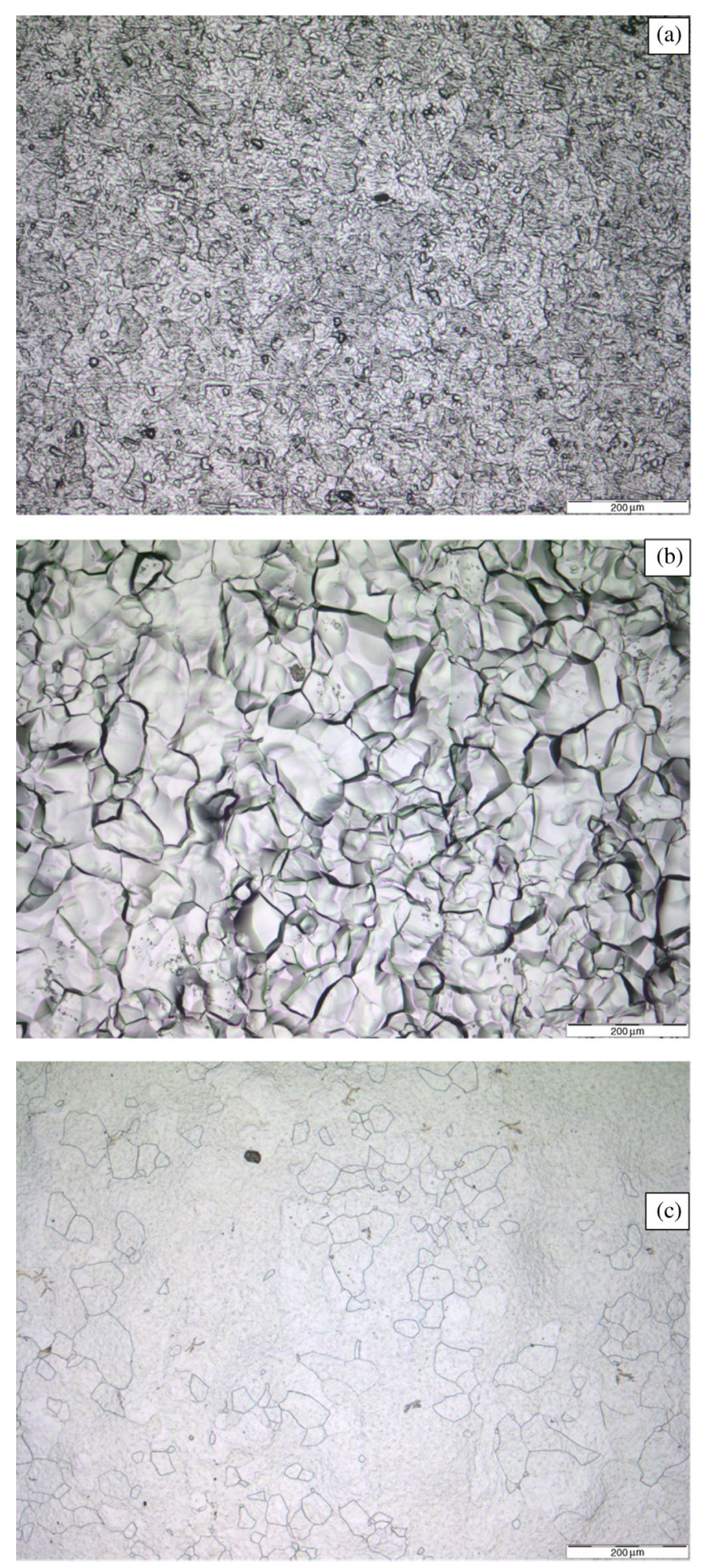

FIG. 1. Optical images of niobium sheet (a) as received, (b) after BCP, and (c) after EP. The white bar at the lower right of each image corresponds to $200 \mu \mathrm{m}$. Optical microscopy is courtesy of Thomas Kiederowski, Institute for Laser Technology, Aachen. smoothness, $\mathrm{BCP}$ is now being replaced by electropolishing (EP) in a typically flowing 1:10 mixture of hydrofluoric and sulfuric acids at $20^{\circ} \mathrm{C}-35^{\circ} \mathrm{C}$, with best results obtained at the lower temperatures [9]. While there are exceptions, gradients in excess of $35 \mathrm{MV} / \mathrm{m}$ with a quality factor of about $10^{10}$ are viewed as routinely attainable by EP but not BCP. Figure 1 shows optical images of typical surfaces as received and after etching.

In these images, it is evident that the surface of the asreceived material is significantly transformed by either $\mathrm{BCP}$ or EP. BCP shows prominent grain boundaries with smaller hillocks or facets within. EP shows only moderately delineated grain boundaries. Topography at a dimensional scale smaller than several microns cannot be discerned at this magnification.

\section{CHARACTERIZATION AND METHODOLOGY}

\section{A. Topography characterization}

Crediting as valid the notion that "smoother is better," there is need for a clear definition of smoothness and its measurement as it relates to SRF performance and cavity processing. Surface topography is routinely measured by stylus profilometry and atomic force microscopy (AFM). In either case, a probe is moved to a series of locations along a line on the surface and its vertical position is measured. The difference between the vertical position at any specific point and the average vertical position of all points can be computed. Its root mean square (RMS) value, $R_{q}$, is one of the simplest descriptions of roughness [10]. The referenced standard indicates a scan length over which $R_{q}$ should be measured. It is not appropriate for the niobium materials, which have an inherent potential nonuniformity at the scale of the grain size, 50-100 $\mu \mathrm{m}$ here. Another root mean square (RMS) value $R_{d q}$ is used to measure slope fluctuation, the $R_{d q}$ is defined by slope angle of six adjacent points in [11] as

$$
\begin{gathered}
R_{d q}=\sqrt{\frac{1}{N} \sum_{i=1}^{N} \Delta_{i}^{2}} \\
\Delta_{i}=\frac{1}{60 d x}\left(y_{i+3}-9 y_{i+2}+45 y_{i+1}-45 y_{i-1}+9 y_{i-2}-y_{i-3}\right),
\end{gathered}
$$

where $y_{n}$ are the height of adjacent points, $d_{x}$ is the step length, and $N$ is the total number of points.

Studies of BCP-treated niobium reported typical roughness values of $1.6 \mu \mathrm{m}$, with a standard deviation of about $0.2 \mu \mathrm{m}$ [12]. The same researchers reported values for EP of $0.1-0.3 \mu \mathrm{m}$, depending on specifics. Further insight can come from viewing the distribution of vertical displacements from the average. Figures 2 and 3 present the height histogram and the AFM scan profile of typical BCP and EP 


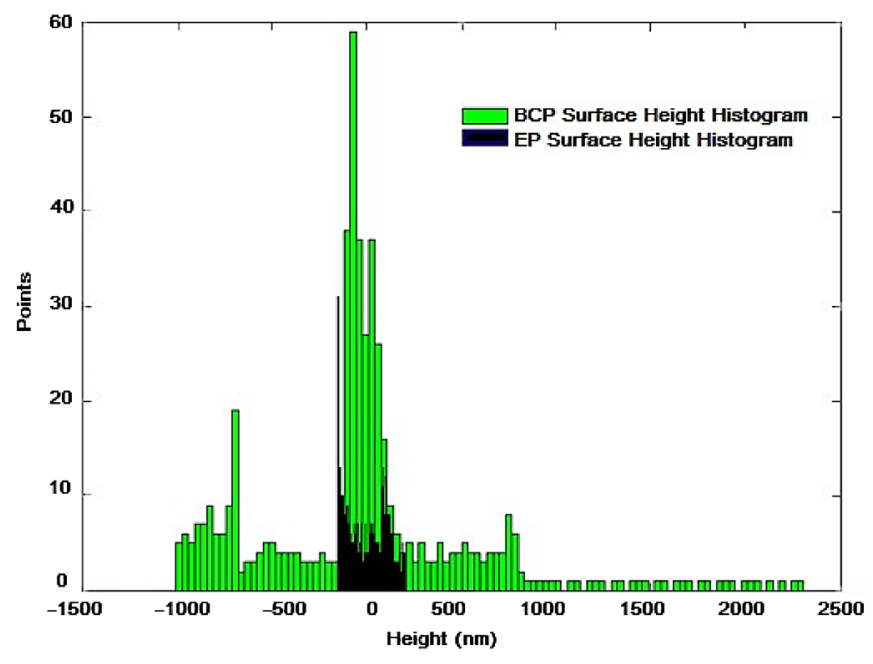

FIG. 2. Frequency distribution of height difference from mean after BCP (100 $\mu \mathrm{m}$ removal) or EP (50 $\mu \mathrm{m}$ removal) both at $20^{\circ} \mathrm{C}$. The total scanning point number is 262144 . The bin for $\mathrm{BCP}$ is $25 \mathrm{~nm}$ and bin width for EP is $5 \mathrm{~nm}$.

surfaces. The BCP samples have more height range than do the EP samples.

The value of average roughness can be strongly impacted by the lateral distance over which data are collected. A previously reported comparison of niobium SRF materials treated in different ways found that $R_{q}$ values increased more than fourfold as the sampled area increased from $20 \times 20 \mu \mathrm{m}$ to $1000 \times 1000 \mu \mathrm{m}$ [12]. Use can be made of the variation of $R_{q}$ with scan length to acquire lateral information [13]. For the present materials, it may be expected that scan lengths less than the grain size will tend to reflect intragranular information, while longer will include the grain boundaries as well, as seen below.

A further way to view AFM or stylus profilometer data is to decompose the whole scan into segments of chosen

(a)

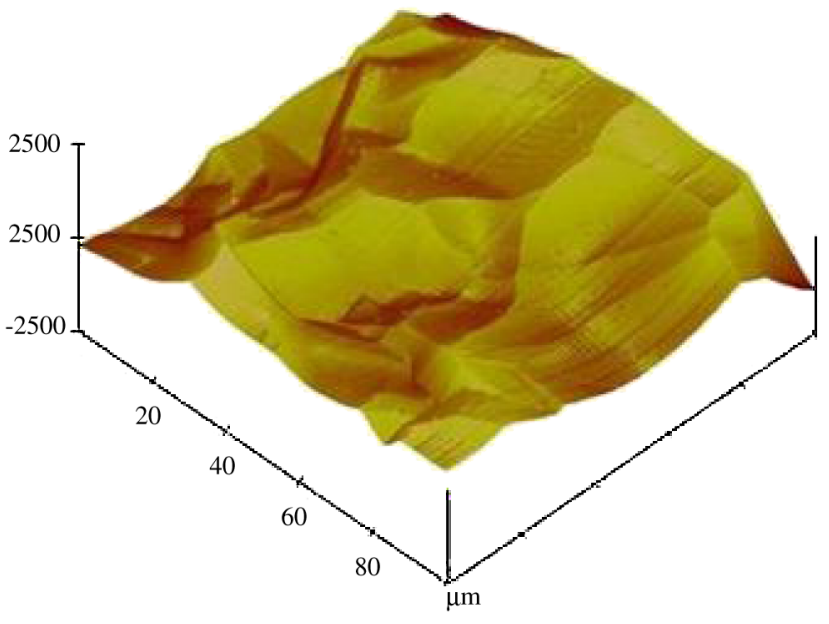

length and then determine $R_{q}$ for each segment. The likelihood of including (e.g.) a grain boundary increases with segment length, and the slope in Fig. 4 shows this proportionality up to some lateral length. Once the segment length reaches a value where the number of grain boundaries per unit length is substantially constant, the curve flattens considerably. The notable feature in this data is that after electropolishing at $-30^{\circ} \mathrm{C}$, essentially no dependence on segment length is seen, providing evidence of nearly complete leveling.

Similar to the electropolished surface, the results from BCP in Fig. 5 indicate that there is a saturated $R_{q}$ at certain scanning length. This distance, larger than which the topography values are substantially constant, may be defined as the correlation length. Visual inspection suggests a value a little less than $80 \mu \mathrm{m}$, consistent with the 50-100 $\mu \mathrm{m}$ grain size. The correlation length can be more precisely determined by use of an autocorrelation function (ACF) [14]. Defining $h(x)$ as the difference between the height value at $x$ from the average value, ACF can be defined as

$$
\operatorname{ACF}(L)=\int_{0}^{L} h(x) h(x+L) d x,
$$

where $L$ is a fixed distance added to all values of $x$ and $h(x)$ is the profile function. The highest value of $L$ for which the value of the integral is constant is the correlation length. At the correlation length (CL), the value of the ACF falls toward zero. An appropriate scan length for ACF determination 3-4 times its expected $75 \mu \mathrm{m}$ value, here about $200 \mu \mathrm{m}$, not the $100 \mu \mathrm{m}$ scan length limit of our AFM. Calculating the ACF from data sets in Fig. 5 gives a CL value around $30 \mu \mathrm{m}$, noticeably too small. Typically, correlation length is proportional to grain size, $20-50 \mu \mathrm{m}$ in fine-grained $\mathrm{Nb}$. Therefore, measuring it requires at least a $200 \mu \mathrm{m}$ scan length, preferably 3 to 4 times the correlation length.

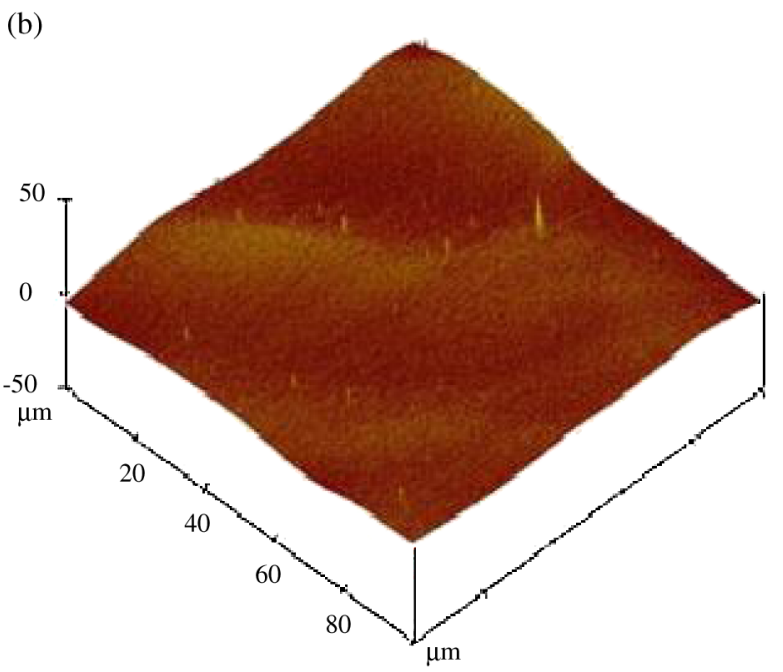

FIG. 3. Topography images created from AFM data (a) after BCP and (b) after EP treatment, the same samples as in Fig. 2. The horizontal scales are both $100 \mu \mathrm{m}$ by $100 \mu \mathrm{m}$ and the vertical scales are 2500 and $50 \mathrm{~nm}$, respectively. 

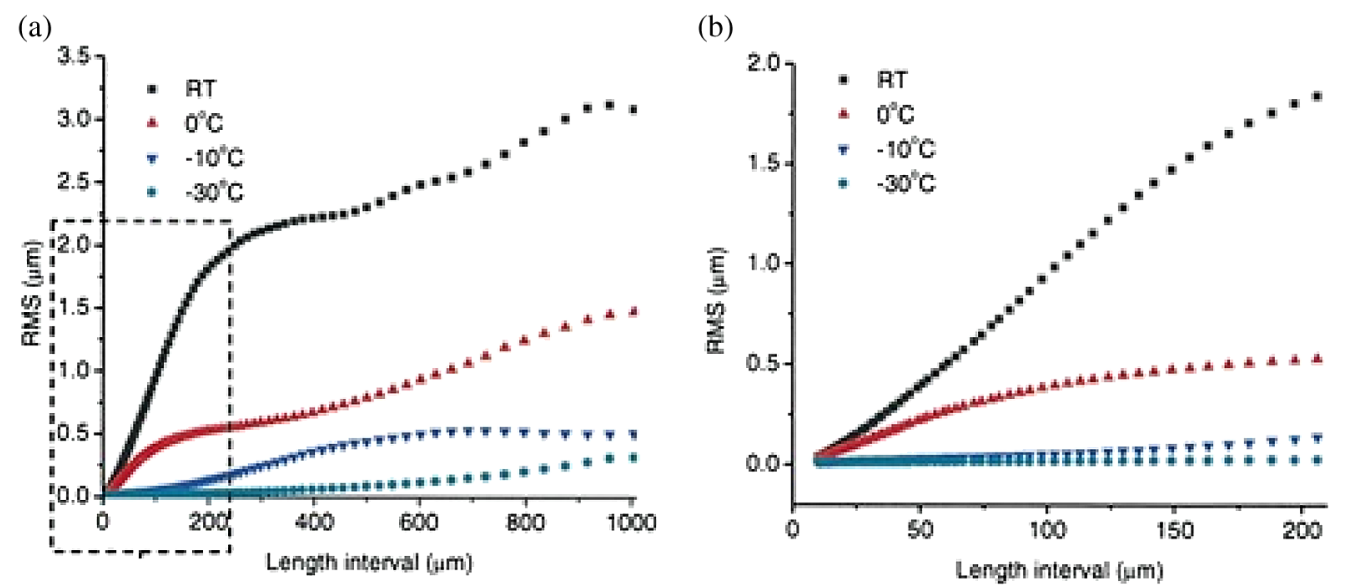

FIG. 4. Stylus profilometry measurements of $R_{q}$ of niobium sheet electropolished in $2 \mathrm{~N}$ sulfuric acid in methanol at the indicated temperature [40].

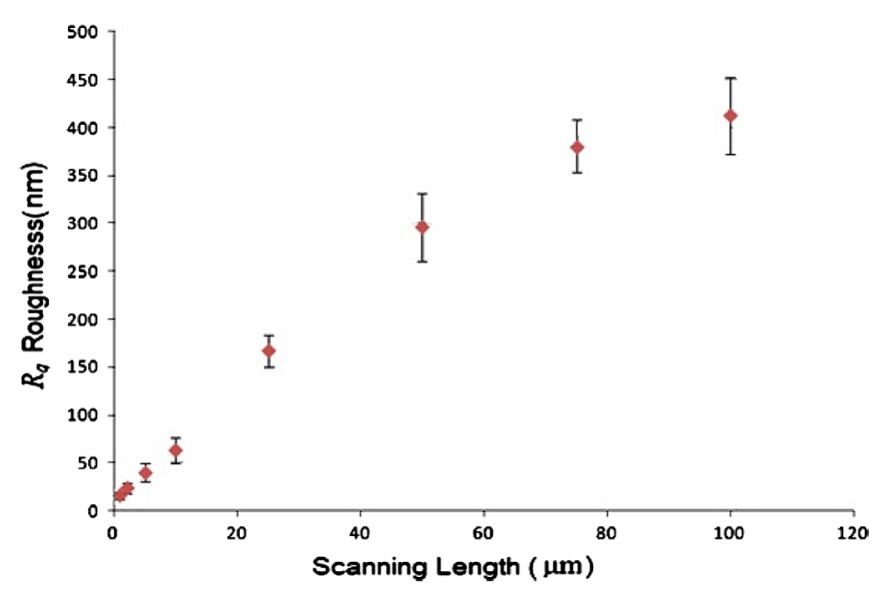

FIG. 5. $R_{q}$ as a function of AFM scan length for $\mathrm{Nb}$ after $100 \mu \mathrm{m}$ removal by BCP, average of three samples.

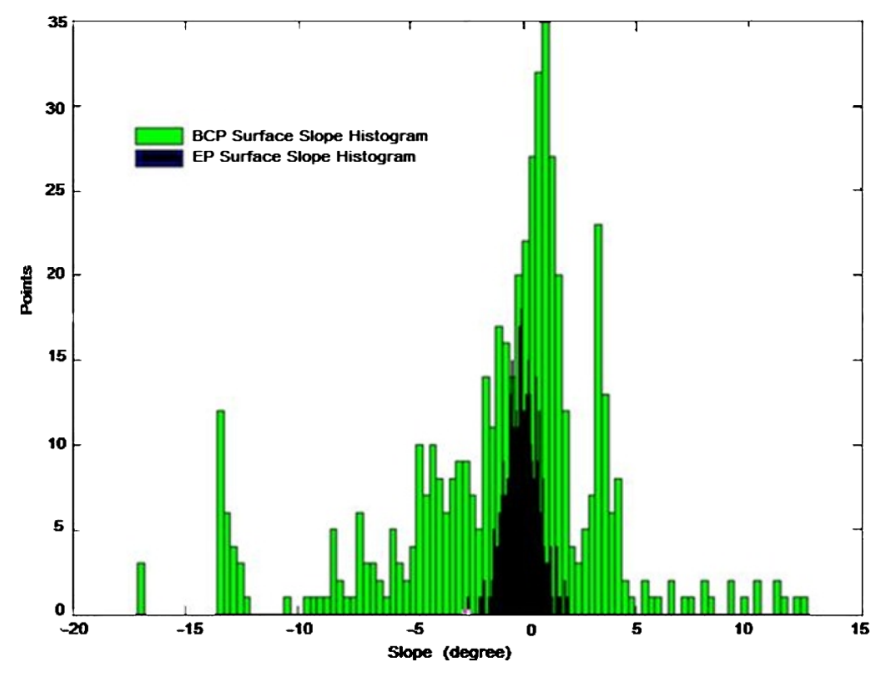

FIG. 6. Slope histogram of the scans in Fig. 2. The total scanning point number is 262144. The bin for BCP is $0.25^{\circ}$ and bin width for EP is $0.05^{\circ}$.
Deeper insight into topography can be obtained by considering the variation in vertical position between adjacent measurements, expressed as slope, to capture the notion of sharp features. Histograms are usually used to determine the structure of surface, revealing isolated features such as holes or bumps. Figure 6 is the slope histogram of scans of $\mathrm{Nb}$ surfaces treated by BCP or EP.

The greatly increased frequency in the number of highslope points for BCP reflects what is qualitatively evident in the optical images earlier. It needs to be understood that the value of slope is affected by the distance between adjacent points. Spacing the points more closely would result in higher slope values for very sharp features, the very ones thought to be most harmful for high-field SRF cavity performance. Further, the sampling issue noted earlier still applies-collecting data from a larger area increases the likelihood that a point of still higher slope will be detected. Finally, the "fatal flaw" impacting SRF performance is likely to be an extreme point, such as the most prominent sharp projection. From that perspective, the distributions such as Figs. 2 and 6 may be more informative than the various averages. All the statistical methods above are limited by the scan length and characterization resolution, and give no roughness information at different scales.

\section{B. Power spectral density (PSD) data analysis}

The optics and the microelectronics communities encountered the need to deal more incisively with topography data some years ago [14]. They found it fruitful to make a Fourier transform of the scanning probe topography data to obtain the contribution at different lateral scales: the power spectral density (PSD). Sharper features will have a more relative contribution at shorter scales as compared with gentle undulations of the same vertical amplitude, for example.

Power spectral density has been used as a tool to combine measurements from different scales and different 
instruments $[12,14,15]$. It represents the spatial-frequency spectrum of surface roughness measured in inverse-length units. The power spectral density function of a surface profile $h(x, y)$ is defined as $[12,14,15]$

$$
\begin{aligned}
& \operatorname{PSD}\left(f_{x}, f_{y}\right) \\
& \quad=\lim _{L \rightarrow \infty} \frac{1}{L^{2}}\left|\int_{-L / 2}^{L / 2} \int_{-L / 2}^{L / 2} h(x, y) e^{-2 \pi i\left(f_{x} x+f_{y} y\right)} d x d y\right|^{2},
\end{aligned}
$$

where $f_{x}$ and $f_{y}$ are the rectangular components of surface frequencies. From Eq. (4), we know that the PSD gives information about the relative contributions of all the possible surface spatial frequencies for an ideal measurement of an infinite surface in the limiting case from 0 frequency (an infinite surface) to an infinite frequency (infinitely small structure) [14]. PSD represents the squared amplitude of surface features plotted against the spatial frequency of those features. PSD and ACF are Fourier transform pairs.

In practice, topographic images of surfaces are recorded in the form of digitized data of surface height, which is finite rather than infinite and sampled rather than continuous. The PSD for digitized data in one dimension used in this study is defined by Eq. (5) [14],

$$
\operatorname{PSD}\left(f_{x}\right)_{N}(m)=\frac{\Delta x}{N}\left|\sum_{n=0}^{N-1} h(x)_{n} \exp (-i 2 \pi n m / N)\right|^{2} K(m),
$$

where $N / 2 \leq m \leq(N / 2)-1$. Equation (5) gives an expression for the $m$ th term in the PSD calculated from a profile of $N$ points; it gives PSD amplitude in units of length cubed. There are discrete values of $f_{x}=m / L$, where $L$ is the measurement length and $x$ in the function of $h(x)_{n}$ takes on discrete values: $x=(L / N)_{n}$. Also, $\Delta x$ is the spacing between data points in the profile, $h(x)_{n}$ are the height values of the profile data points, and $K(m)$ is a bookkeeping factor that equals 1 except that $K( \pm N / 2)=$ $1 / 2$ at the ends of the power spectrum. More complete mathematical descriptions can be found in Refs. [14,15].

A very important connection between $R_{q}$ and PSD is that one can calculate the $R_{q}$ distribution over a specific frequency range. The contribution from that scale is a portion of total roughness composition of the whole scan range. Note that integration of the PSD yields the square of $R_{q}$ over the range of integration [15],

$$
\left.\left(R_{q}\right)^{2}\right|_{f_{0} \rightarrow f_{1}}=\int_{f_{0}}^{f_{1}} \operatorname{PSD}(f) d f .
$$

Four major limitations are involved in PSD calculations from real data [16]: (i) bandwidth limits, (ii) aliasing, (iii) trending, and (iv) statistical instability [17]. In this study, the bandwidth limits of surface profile measurements are determined by the total trace length, sampling interval, and various filtering operations. The surface frequency limits included in this study are [18]

$$
f_{\min }=\frac{1}{L}, \quad f_{\max }=f_{\text {Nyquist }}=\frac{N}{2 L},
$$

where $L$ is total trace length sampled at $N$ equally spaced points. In practice, bandwidth limits may be extended by acquiring further scans with greater or lesser distance between data points. The issue of a possible difference in locations scanned needs to be considered.

Any instrument that acquires topography data convolutes its own signature with the data, the machine transfer function, where "aliasing" is a harmful effect. Antialiasing of stylus profilometry is accomplished by introducing a low pass filter for surface frequencies greater than the Nyquist frequency $[19,20]$ :

$$
\bar{M}(f)=\frac{1}{1+\left(f d_{0}\right)} .
$$

The value of $d_{0}$ is optimized from tip scanning speed, and defined as the spatial wavelength for $50 \%$ amplitude attenuation of this low pass filter. We chose to use $d_{0} 0.862 \mu \mathrm{m}$ as suggested by Ref. [19] for a stylus profilometer scan with resolution of about $0.65 \mu \mathrm{m}$.

In order to explore well the fine scale structure evolution with different treatments and avoid a spurious trend which might be due to the presence of surface components with wavelengths longer than the record length [21], a background must be removed from all data to effectively flatten the mean surface- "detrending." Experience in the optics and semiconductor communities indicates that a suitable approach is to remove a two-dimensional third order polynomial from each record before further analysis [18,22]. Such an approach is promising for relatively smooth surfaces (EP), but major sharp surface steps (BCP) may need to be individually removed- "destepping."

Windowing is used to eliminate the discontinuity at the two end points, which may otherwise cause error while calculating the Fourier transform [20]. With a window function, the surface fractal property will be correctly shown. In addition, in order to reduce the statistical instability of real data and minimize the measurement errors, a standard way of stabilizing the PSD function is used: averaging. Figure 7 below shows PSDs calculated after only averaging data. Figure 8 shows PSD's calculated after additionally addressing antialiasing, detrending, and windowing.

For the data shown in Figs. 7 and 8, power spectra for all traces in the fast scan direction were averaged, and the PSD profiles measured at different locations under the same scan condition were also averaged. A Tukey window transform was applied in Fig. 8 in order to eliminate spurious high-frequency noise and maintain the amplitude of $R_{q}$ [23]. As shown in the comparison of Fig. 7 to Fig. 8, the surface fractal property will be correctly shown with use of 


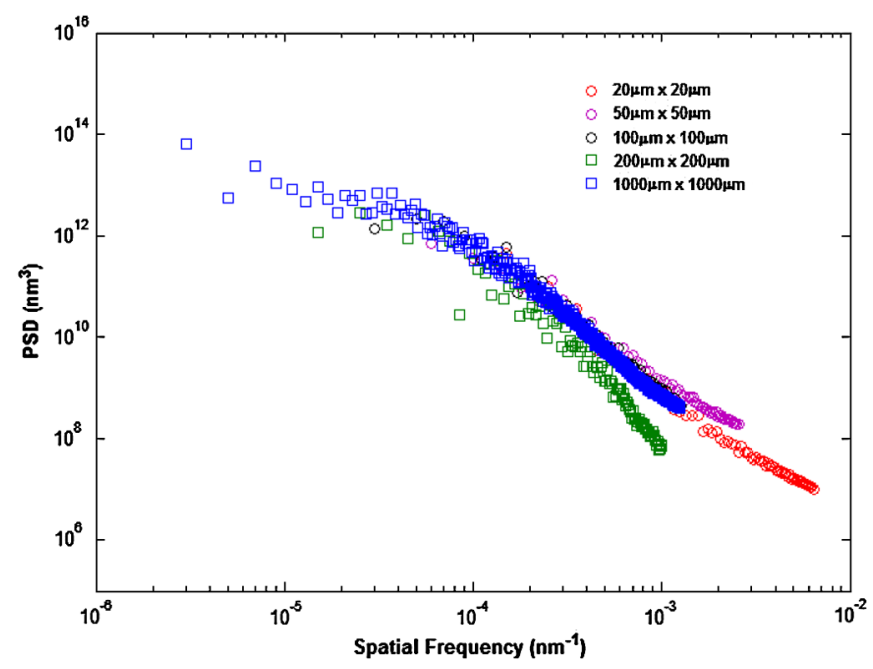

FIG. 7. Power spectral density calculated directly from the raw data for the indicated scans collected from fine-grained niobium sheet subjected to $5 \mathrm{~min}$ of BCP.

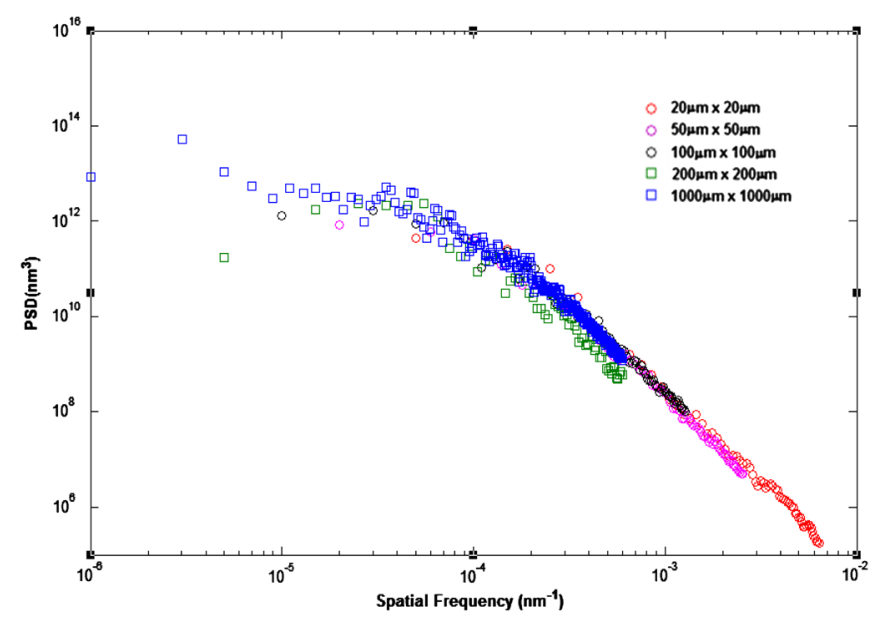

FIG. 8. Power spectral densities calculated from the same data as Fig. 7, but with the corrections discussed in the text.

a window function. In order to reduce the impact of the window function on the averaged PSD, a further polynomial-based deconvolution is conducted in MATLAB ${ }^{\circledR}$ code [24].

The impact of proper data treatment on the opportunity to combine different data sets is clearly evident by comparison with previous PSD calculation in Fig. 7 [25]. PSDs in Fig. 8 correct artifacts such as the high-frequency leveling and the middle frequency "peak." Certain characteristic features of such PSD data from Nb BCP samples are evident: (i) power falls with increasing frequency; (ii) a significant data range at high frequencies is a straight line on the $\log / \log$ plot indicating a power law function; and (iii) in the lower frequency data range, the frequency dependence of power departs from linear and approaches a constant value.
To summarize, for stylus profilometry or atomic force microscopy, tip size, spacing of points, number of points, and machine characteristics limit precision, and contribute artifacts, obtaining an accurate PSD of a surface requires a series of steps: (i) data acquisition; (ii) antialiasing correction: to reduce contribution from machine transfer function, tip size; (iii) detrending correction: to reduce contribution from surface curvature-lack of planarity at large scale; (iv) windowing correction: to reduce artifacts on transform due to finite length; (v) averaging: to reduce the statistical instability of real data.

\section{Components analysis}

Researchers [22,26,27] in the optics and microelectronics communities applying PSD analysis have noticed characteristic patterns associated with frequently occurring topographies. For example, a $90^{\circ}$ sharp step gives a PSD having the form $K / f^{2}$, a straight line of slope -2 in log-log coordinates. PSD data may be further analyzed in terms of characteristic patterns. Such structures may frequently be described in terms of three idealized models, fractal structure, and two superstructure models.

\section{Fractal structure model}

As is evident in Fig. 8, the high-frequency portion of the PSD plot for BCP-treated niobium surface appears as a straight line on a $\log / \log$ plot and so can be fit by a power law expression having the form

$$
S(f)=K_{n} / f^{n},
$$

where $K_{n}$ and $n$ are the fitting parameters. Note $n$ called fractal dimension, is often between 2 to 3 , and larger $n$ means rougher surface. Power law forms arise from fractal surface topography inter alia, a self-affine topography that is self-similar, having key features repeating at successive dimensional scales, schematically depicted as a Koch curve shown in Fig. 9.

Characteristic fractal structures are associated with specific values of the fractal dimension $D$, given by $D=$ $(7-n) / 2$. Integrating the expression for $S(f)$ yields the fractal structure $R_{q}$ and correlation length CL,

$$
R q=\left[\frac{K_{n} L^{n-1}}{n-1}\right]^{1 / 2}, \quad \mathrm{CL}=\frac{(n-1)^{2} L}{2(2 n-1)}
$$

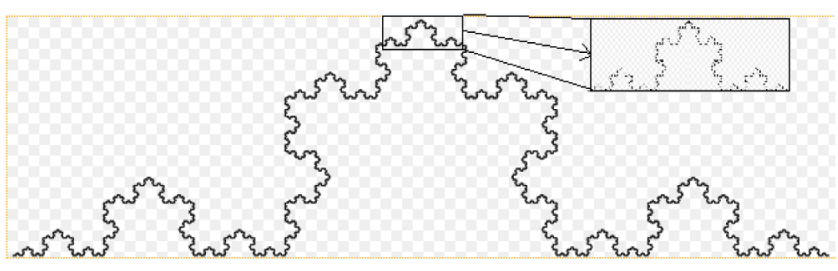

FIG. 9. Koch curve illustration of fractal structure [41]. 
where $K_{n}$ is the scaling factor, $n$ is the fitting order, and $L$ is the scan length $[28,29]$. Note, however, that the correlation length obtained from the above equation is necessarily bounded by the scan length.

\section{Superstructure models}

$$
\text { a. "K correlation" or "ABC Model” [30-33] }
$$

Structure present at a larger dimensional scale, such as grain size in the present case, may be superimposed on small-scale structure. Such structure has been described in cluster ion beam polishing and in thin film growth [22]. The PSD of such a model is described by

$$
S(f)=A /\left[1+(B f)^{2}\right]^{(C+1) / 2} .
$$

$A, B$, and $C$ are fitting parameters, with $C>1$. It is possible for a surface to have superstructure at more than one scale, each described by such an expression. For such a model, one obtains $R_{q}$ and CL [26]:

$$
R_{q}^{2}=\frac{2 \pi A}{B^{2}(C-1)}, \quad \mathrm{CL}^{2}=\frac{(C-1)^{2} B^{2}}{2 \pi^{2} C}
$$

Note that this calculated $R_{q}$ and $\mathrm{CL}$ refer to the $K$-correlation contribution only.

\section{b. Shifted-Gaussian model [34]}

The shifted-Gaussian model basically described a surface with isolated islandlike structures. Rasigni found this in a PSD with structure that fits a Gaussian distribution but with peak shifted [34]. The model gives clear definitions of size, height, and periodicity of particles on an otherwise independently structured surface, which in our case can help calculating local $R_{q}$ and CL. It has been demonstrated suitable for describing the initial stage of thin film growth models [34]. A shifted-Gaussian component to surface roughness contributes to the PSD as

$$
S(f)=\pi \sigma^{2} \tau^{2} e^{\left[-(f-x)^{2} \tau^{2} \pi^{2}\right]},
$$

where $\sigma, \tau$, and $x$ denoted the height, size, and periodicity of superstructures.

For example, the PSD of a BCP-treated surface might consist of a shifted Gaussian at low frequencies reflecting the grain structure and mostly a straight line at higher frequency reflecting the prominent sharp edges. In contrast, PSD data obtained from electropolished materials typically do not exhibit straight lines on a log/log plot but display two regions of strong curvature, as will be seen later. More generally, the PSD of a niobium surface prepared by various methods can be fruitfully decomposed into contributions from each of these types of models [22,26,35-37]:

$$
\begin{aligned}
\mathrm{PSD}(\text { total })= & \mathrm{PSD}_{\text {fractal }}+\mathrm{PSD}_{K} \text { correlation } \\
& +\mathrm{PSD}_{\text {shift Gaussian }} .
\end{aligned}
$$

One may optimally fit the measured PSD curves with such components by using the Levenberg-Marquardt least squares method within the chosen frequency regions. Usually, a certain PSD pattern can dominate within a certain frequency range. Thus, one may fit the PSD in this frequency range with only that dominant model. Accomplishing the fit proceeds with awareness of the characteristic forms arising from the surface processing that has been employed, as indicated above, to make an initial guess. Automatic fitting procedures can then be employed to converge on a best fit. Having these fitting parameters, one can derive the physical parameters: fractal dimension, $K$ correlation $R_{q}$ and correlation length, and particles' size, height and frequency, etc., and learn the surface evolution during process.

\section{APPLICATIONS AND RESULTS}

We illustrate with three sets of materials. (i) Single crystal $\mathrm{Nb}$ materials were obtained by cutting from a largegrained ingot; the surface orientation was determined by electron backscatter diffraction to be $\langle 110\rangle$. (ii) Finegrained $\mathrm{Nb}$ was standard residual resistivity ratio sheet material used for cavity production. Both of these types of samples were polished to great smoothness (nanopolished) by a proprietary treatment (Wah Chang). While the vendor does not disclose the details of their method, we are able to obtain substantially similar results by careful use of metallographic polishing methods. Samples of each type were subjected to $1: 1: 2 \mathrm{BCP}$ at $10^{\circ} \mathrm{C}$ to achieve the material removals indicated later. (iii) Some fine-grained samples were subjected to EP after $100 \mu \mathrm{m}$ removal by BCP.

Topography was determined by AFM. $100 \mu \mathrm{m}$ by $100 \mu \mathrm{m}$ areas on each were examined by AFM. AFM

TABLE I. Averaged $R_{q}$ and $R_{d q}$ parameters with and without third order polynomial detrending with AFM scan range $100 \mu \mathrm{m}$ by $100 \mu \mathrm{m}$ (unit: $\mathrm{nm}$ ).

\begin{tabular}{lcccccc}
\hline \hline Samples & \multicolumn{2}{c}{ Single crystal 13} & \multicolumn{2}{c}{ Nanopolished fine-grained 10 } & \multicolumn{2}{c}{ "Standard" fine-grained } \\
Treatment & As received & After 30 $\mu \mathrm{m} \mathrm{BCP}$ & As received & Initial $(5-6 \mu \mathrm{m}) \mathrm{BCP}$ & $100 \mu \mathrm{m} \mathrm{BCP}$ & $50 \mu \mathrm{m} \mathrm{EP}$ \\
\hline$R_{q}(\mathrm{~nm})$ & 14 & 13 & 53 & 155 & 458 & 72 \\
$R_{q}(\mathrm{~nm})$ detrended & 11 & 10 & 16 & 148 & 337 & 47 \\
$R_{d q}\left({ }^{\circ}\right)$ & 0.67 & 1.1 & 0.38 & 3.8 & 4.0 & 0.95 \\
$R_{d q}$ detrended $\left(^{\circ}\right)$ & 0.67 & 1.1 & 0.35 & 3.8 & 3.9 & 0.93 \\
\hline \hline
\end{tabular}



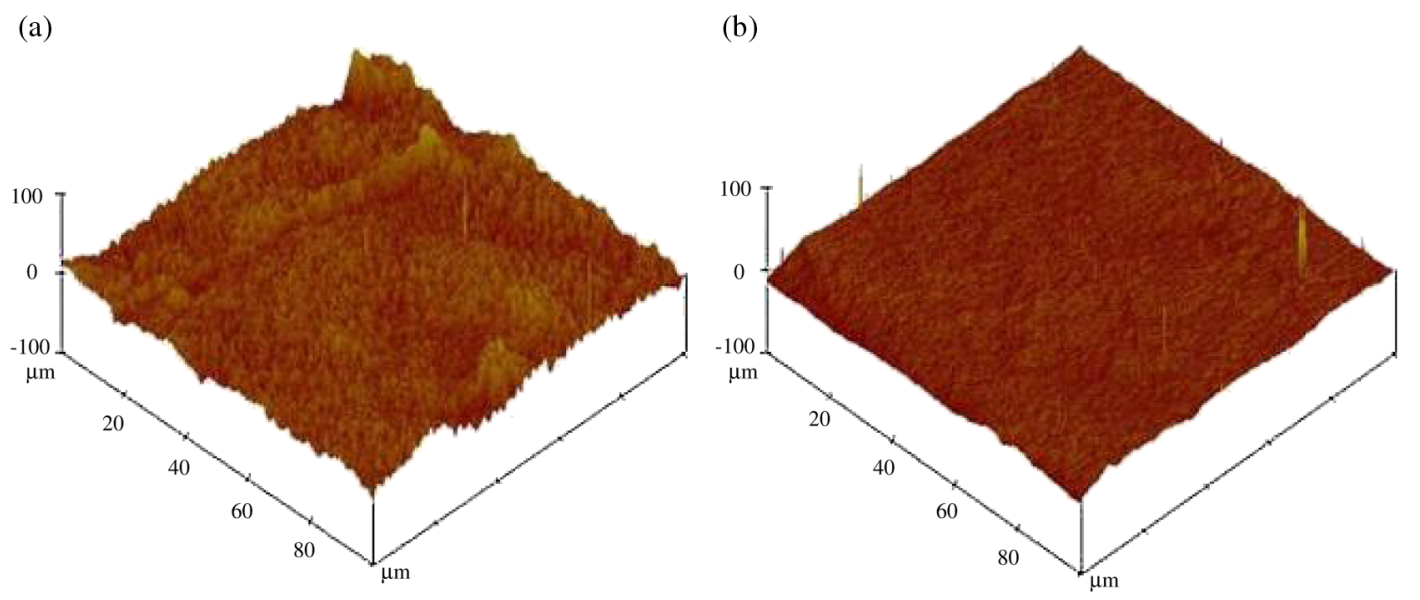

FIG. 10. AFM scans of single crystal sample 13 in Table I: (a) as received and (b) after $30 \mu \mathrm{m}$ removal by BCP. The horizontal scale is $100 \mu \mathrm{m}$ by $100 \mu \mathrm{m}$; the vertical scale is $100 \mathrm{~nm}$.
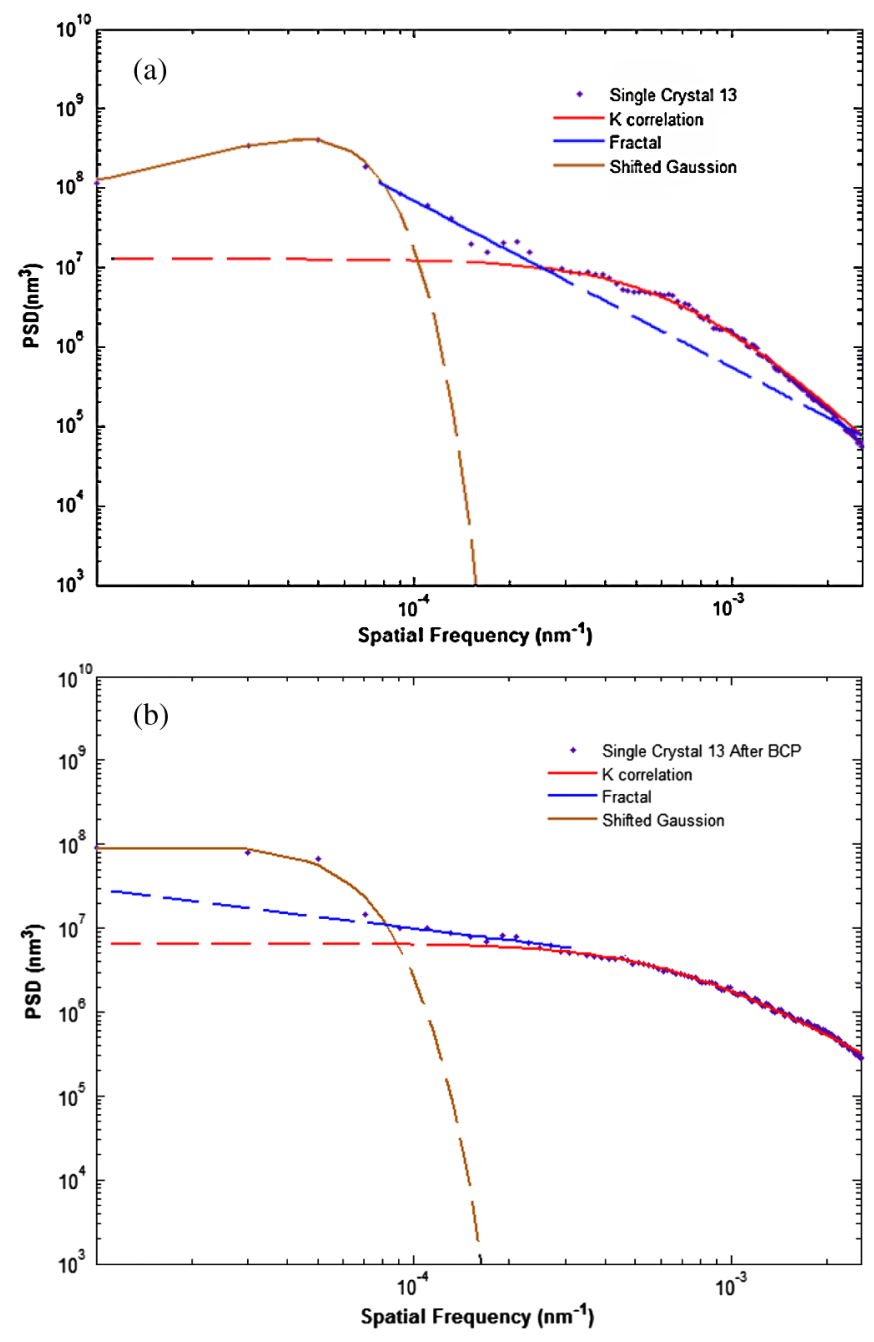

FIG. 11. Average one-dimensional PSD of single crystal 13, (a) as received and (b) after $30 \mu \mathrm{m}$ removal by BCP. The range of fitting for each component is indicated by the solid line. measurements were performed as previously [12] using a commercial AFM (Digital Instruments: Nanoscope IV) in a tapping mode using silicon tips with a diameter of $10 \mathrm{~nm}$. The AFM images were captured as arrays of height values with 512 by 512 points. The $R_{q}$ and $R_{d q}$ parameters with and without detrending were calculated and shown in Table I. Here, the $R_{q}$ and $R_{d q}$ are RMS of height and slope angle. Notice that the $R_{q}$ value decreases after detrending but $R_{d q}$ is almost unaffected.

\section{A. Single crystals}

Since grain boundaries are absent, single crystal samples may correspond to the polishing of grain interiors in finegrained materials. Four single crystal samples with unknown prior history were characterized before and after $30 \mu \mathrm{m}$ BCP etch. AFM scans were performed on four different locations on each sample. Representative scans are presented in Fig. 10.

Surfaces in Fig. 10 give an $R_{q}$ change from 11 to $10 \mathrm{~nm}$ in Table I, while Fig. 10 illustrates that the surface becomes significantly smoother through BCP etching. Even though this visual impact is significant in the AFM image, $R_{q}$ almost remains the same. More insight is required for an incisive analysis.

\section{PSD results and analysis}

The average 1D PSD derived from the AFM scans of Fig. 10 are shown in Figs. 11(a) and 11(b) to compare the single crystal sample before and after the BCP process. They may be fit by a fractal model $+K$-correlation model + shifted-Gaussian model. Three components are fitted in Fig. 11; fitting parameters are given in Table II.

Three components are fit in Fig. 11, fitting parameters are given in Table II. 
TABLE II. Fitting parameters of samples (unit: nm) for Fig. 11.

\begin{tabular}{|c|c|c|c|c|c|c|c|c|}
\hline \multirow[b]{2}{*}{ Model: (nm) } & \multicolumn{3}{|c|}{$K$ correlation } & \multicolumn{2}{|c|}{ Fractal } & \multicolumn{3}{|c|}{ Shifted Gaussian } \\
\hline & $A$ & $B$ & $C$ & $K_{n}$ & $n$ & Height $\sigma$ & Size $\tau$ & Frequency $x$ \\
\hline As received & $1.27 \times 10^{7}$ & 1494 & 2.71 & 0.28 & 2.1 & 1.13 & $1.01 \times 10^{4}$ & $4.41 \times 10^{-5}$ \\
\hline After BCP & $6.52 \times 10^{6}$ & 1577 & 1.18 & $1.4 \times 10^{5}$ & 0.47 & 1.25 & $1.10 \times 10^{4}$ & $1.77 \times 10^{-5}$ \\
\hline Frequency range & \multicolumn{3}{|c|}{$2.6 \times 10^{-4}-2.6 \times 10^{-3}$} & \multicolumn{2}{|c|}{$9.0 \times 10^{-5}-2.6 \times 10^{-4}$} & \multicolumn{3}{|c|}{$1 \times 10^{-6}-9.0 \times 10^{-5}$} \\
\hline
\end{tabular}

\section{Discussion}

In Fig. 11, the PSD of the single crystal sample as received shows a straight line at middle frequencies from $9.0 \times 10^{-5}$ to $2.6 \times 10^{-4} \mathrm{~nm}^{-1}$, perhaps due to the previous mechanical polishing history. After BCP etching, the PSD amplitude decreases at low frequency and increases at high frequency. This decrease results in AFM image smoothness, while the increase in high frequency comes from the submicron feature growth. It is apparent that $n$, the fractal dimension, decreases after BCP etching. The PSD amplitude from the fractal component also is reduced by BCP.

\section{B. Initial genesis of BCP topography}

A fine-grained niobium sample received a propriety polish ("nanopolish") by a commercial vendor. We view this treatment as comparable to careful metallographic polishing. The surface topography of these samples was characterized as received, and after 2 min BCP etch at $18^{\circ} \mathrm{C}$. Since we are using 1:1:2 standard BCP solutions, the removal rate is $\sim 3 \mu \mathrm{m} / \mathrm{min}$, so nominally $6 \mu \mathrm{m}$ materials was removed.

\section{Characterization}

Four locations for each state were scanned by AFM. Typical AFM images are presented in Fig. 12.

Figure 12 clearly shows that the sharp features grow and their sizes vary; $R_{q}$ increases from 16 to $148 \mathrm{~nm}$ on these samples. To learn these features profile, one can choose one representative line sectional profile. Figure 13 shows two representative line profiles before and after BCP from the fine-grained nanopolished sample as shown in Fig. 12 by black lines. They illustrate that the grain boundaries emerge in sharp relief.
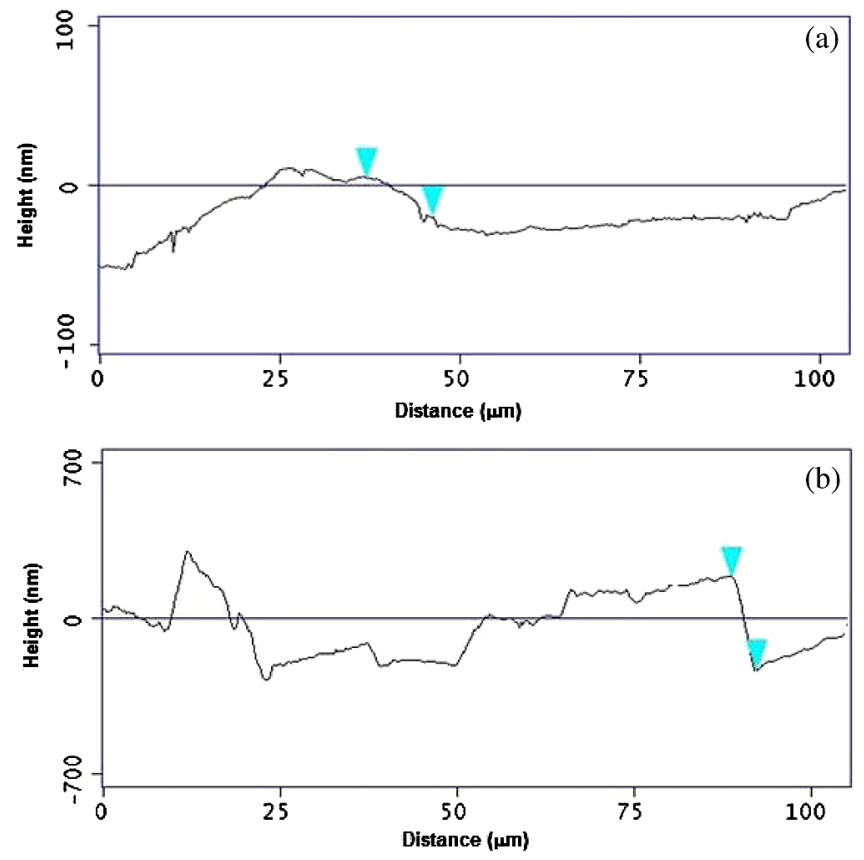

FIG. 13. Surface line profiles of nanopolished fine-grained samples: (a) as received and (b) after $6 \mu \mathrm{m}$ removal by BCP.
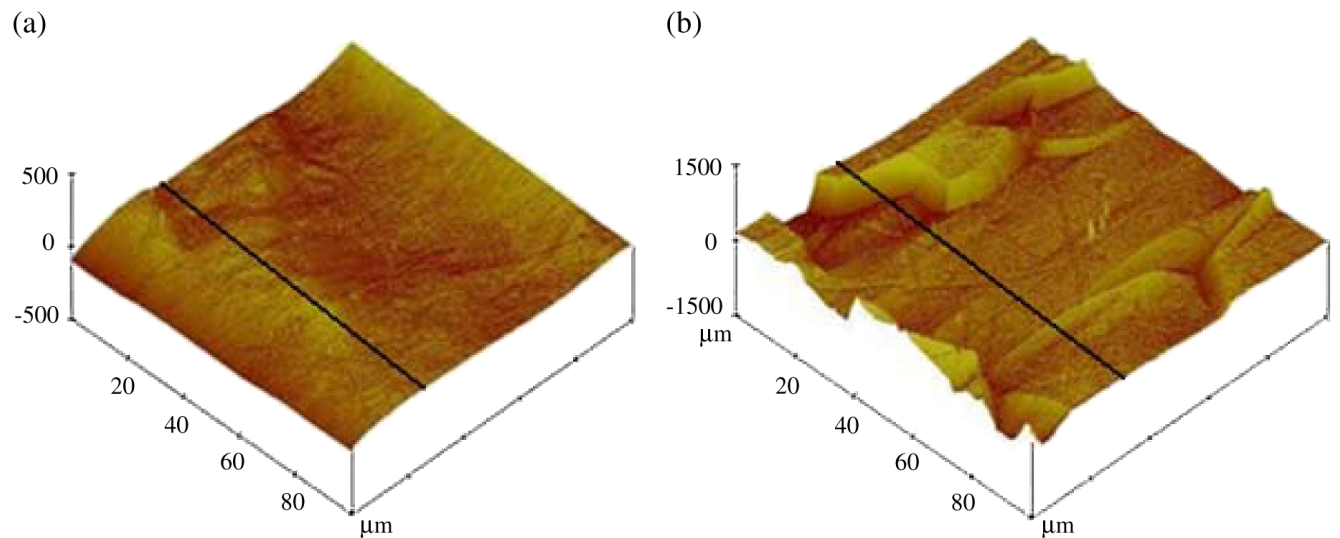

FIG. 12. AFM images of (a) nanopolished fine-grained sample as received and (b) after BCP with $6 \mu \mathrm{m}$ removal. The horizontal scale is $100 \mu \mathrm{m}$ by $100 \mu \mathrm{m}$; vertical scales are 500 and $1500 \mathrm{~nm}$ for (a) and (b), respectively. 
TABLE III. Lateral lengths and angles between the given two cursors shown in Fig. 13.

\begin{tabular}{lcc}
\hline \hline Between two cursors & $\begin{array}{c}\text { Nanopolish } \\
\text { by vendor }\end{array}$ & $\begin{array}{c}2 \text { min BCP } \\
(6 \mu \mathrm{m} \text { removal })\end{array}$ \\
\hline Horizontal distance $(\mu \mathrm{m})$ & 9.2 & 3.5 \\
Vertical distance $(\mathrm{nm})$ & 23 & 415 \\
Angle (degree) & 0.14 & 6.7 \\
\hline \hline
\end{tabular}

Two cursors are placed at potential grain boundary step. Their height difference is peak to valley at the step feature. Note that the vertical scales are different. Length and angle measurements at the marked steps are given in Table III.

From Table III, one observes that the vertical distance grows from 23 to $415 \mathrm{~nm}$, while the horizontal distance remains essentially constant, so that the angle slope of step increases dramatically.

\section{2. $P S D$ results and analysis}

The averaged 1D PSD derived from Fig. 12 AFM scans are shown in Fig. 14 to illustrate the $\mathrm{BCP}$ process on nanopolished samples.

Figure 14 is also regionally fit with different model components and the fitting parameters are given in Table IV.

The PSD of the nanopolished fine-grained sample as received shows a straight line character at midfrequency and superstructure curvature at low and high frequencies, while the PSD of the fine-grained sample after BCP reveals a straight line for the majority frequency range. Presuming that nanopolishing is some variant of chemomechanical planarization, this could reflect random roughening at a very low level together with the signature of the abrasive. But BCP produces differential etching and creates facet surface features, which follows power law character as reflected by the straight line in PSD. In addition, the amplitude of PSD increases substantially around $1 \times 10^{-4} \mathrm{~nm}^{-1}$, this resulting in the $R_{q}$ increasing from 16 to $148 \mathrm{~nm}$.

\section{Discussion}

Previously measured [38] removal rates in 1:1:2 fresh BCP solutions indicate $6 \mu \mathrm{m}$ removal here. In Table III, the increase of greatest height difference from 23 to
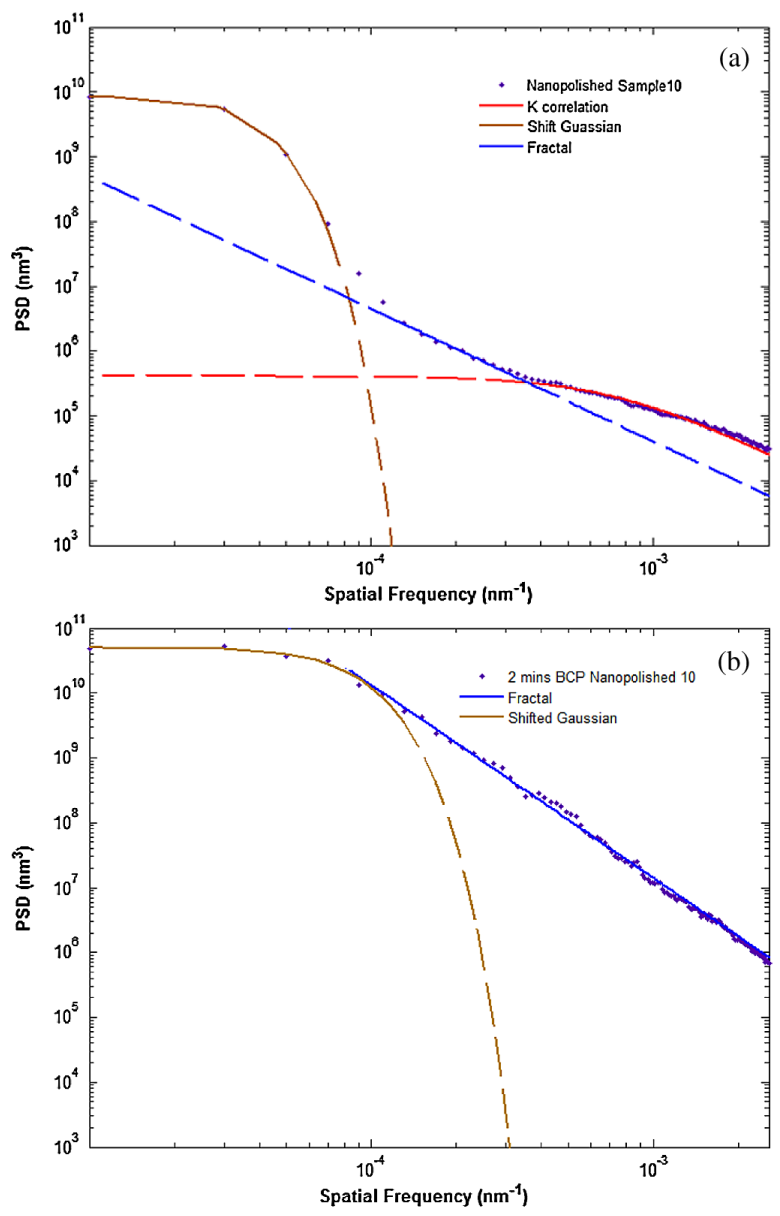

FIG. 14. Averaged 1D PSD for nanopolished fine-grained material: (a) as received and (b) after $6 \mu \mathrm{m}$ removal by BCP. The range of fitting for each component is indicated by the solid line.

$415 \mathrm{~nm}$ at the grain boundary discontinuity suggests a differential etch rate between exposed grain faces of around $200 \mathrm{~nm} / \mathrm{min}$. The lateral length is approximately the observed length of grain boundary incline, so that the slope changes from $0.14^{\circ}$ to $6.7^{\circ}$. Therefore, in the lateral frequency range associated with the grain boundary feature, a one-dimension averaged PSD spectrum amplitude increases as an overall effect of each individual line evolution. Since the intragrain roughness is insensitive to the BCP etching (Table I), its component in PSD increases little.

TABLE IV. The fitting parameter of samples (unit: nm) for Fig. 14.

\begin{tabular}{|c|c|c|c|c|c|c|c|c|}
\hline \multirow[b]{2}{*}{ Model (nm) } & \multicolumn{3}{|c|}{$K$ correlation } & \multicolumn{2}{|c|}{ Fractal } & \multicolumn{3}{|c|}{ Shifted Gaussian } \\
\hline & $A$ & $B$ & $C$ & $K_{n}$ & $n$ & Height $\sigma$ & Size $\tau$ & Frequency $x$ \\
\hline As received & $4.1 \times 10^{5}$ & 1356 & 1.2 & $2.87 \times 10^{-2}$ & 2.05 & 4.35 & $1.20 \times 10^{4}$ & $1.21 \times 10^{-5}$ \\
\hline Frequency range & \multirow{3}{*}{\multicolumn{3}{|c|}{$4.5 \times 10^{-4}-2.5 \times 10^{-3}$}} & \multicolumn{2}{|c|}{$1.2 \times 10^{-4}-4.5 \times 10^{-4}$} & \multicolumn{3}{|c|}{$1 \times 10^{-5}-1.2 \times 10^{-4}$} \\
\hline $6 \mu \mathrm{m} \mathrm{BCP}$ & & & & 0.16 & 2.98 & 27.48 & $4.60 \times 10^{3}$ & $1.73 \times 10^{-5}$ \\
\hline Frequency range & & & & \multicolumn{2}{|c|}{$1.5 \times 10^{-4}-2.5 \times 10^{-3}$} & \multicolumn{3}{|c|}{$1 \times 10^{-5}-1.5 \times 10^{-4}$} \\
\hline
\end{tabular}



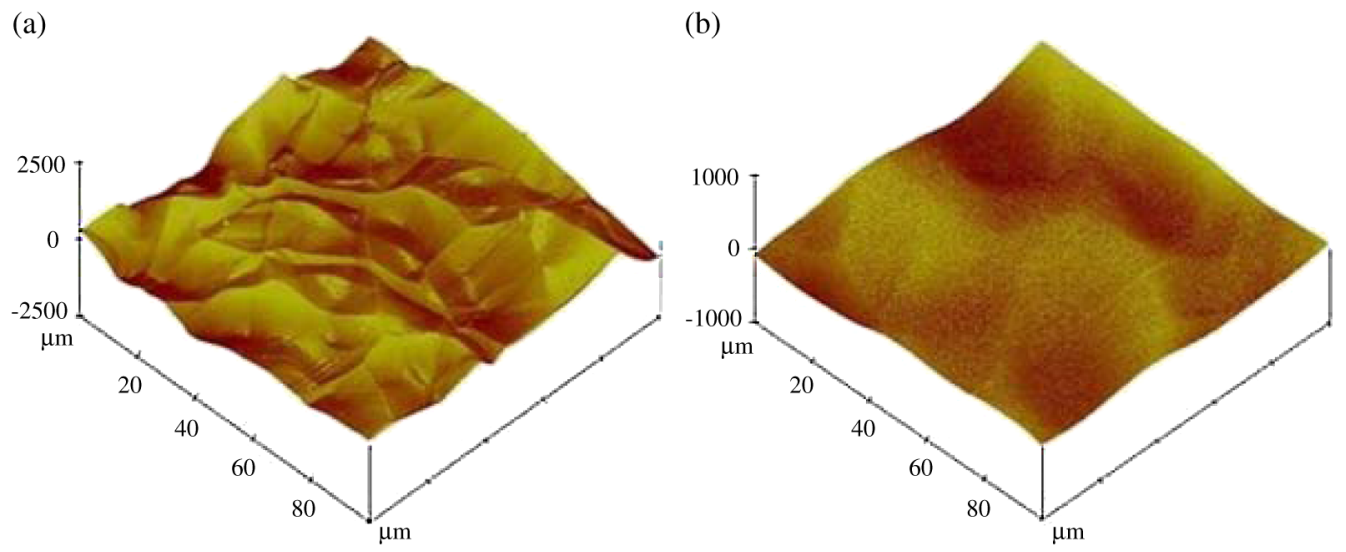

FIG. 15. AFM images of fine-grained material after (a) $100 \mu \mathrm{m}$ removal by BCP or (b) $50 \mu \mathrm{m}$ removal by EP. The horizontal scale is $100 \mu \mathrm{m}$ by $100 \mu \mathrm{m}$ for both. Vertical scales are 2500 and $1000 \mathrm{~nm}$ for (a) and (b), respectively.

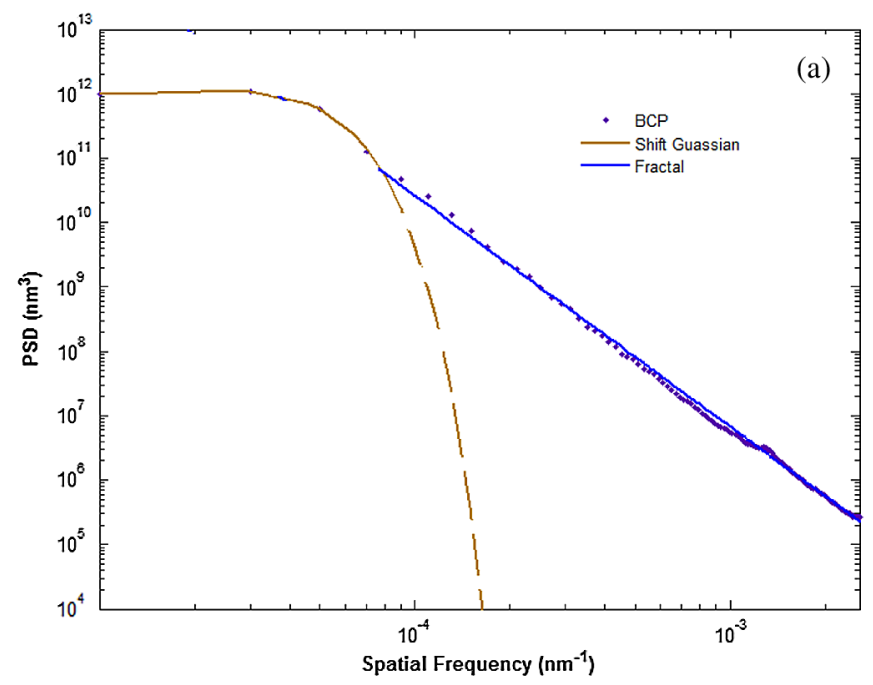

The impact of $\mathrm{BCP}$ is conditioned by the presence of grain boundaries, a candidate for selective attack (see Figs. 12 and 13). The PSD from the nanopolished finegrained $\mathrm{Nb}$ sample shows continuing evolution toward straight line character, reflecting a surface structure dominated by step edges. These grain boundary step edges overwhelm the overall frequency domain. The features of most concern for SRF performance are the pronounced sharp edges at the apparent grain boundaries. On the contrary, in Sec. III A, the single crystals (which do not have grain boundaries) do not evolve toward the same PSD character under BCP treatment. This suggests the need for a characterization approach that discerns a small number of isolated features (e.g. histograms) as well as an integrative approach that discerns the net evolution of surface character.

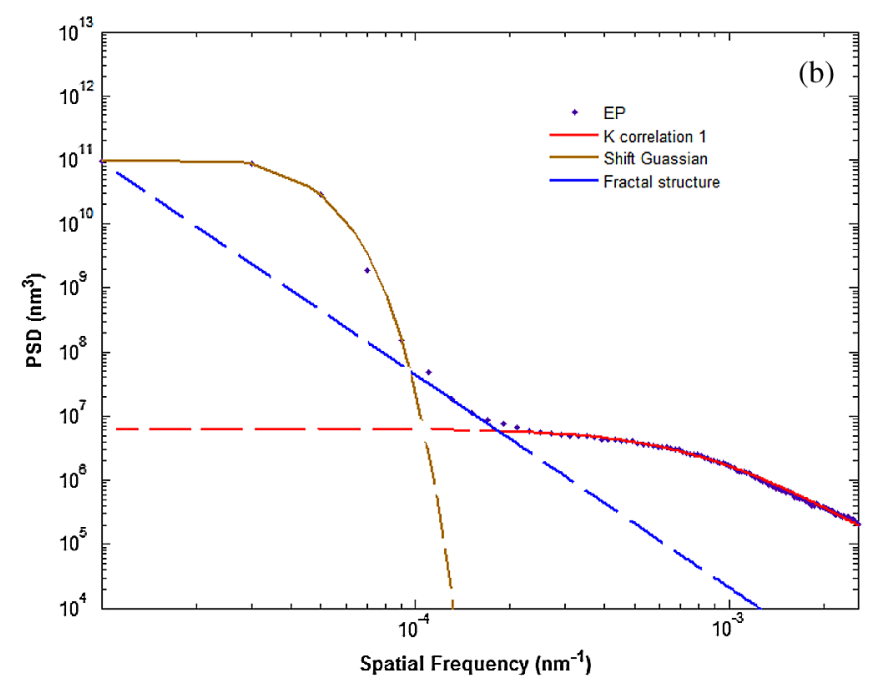

FIG. 16. Average 1D PSD from fine-grained sample after removal of (a) $100 \mu \mathrm{m}$ BCP or (b) after $50 \mu \mathrm{m}$ EP. The range of fitting for each component is indicated by the solid line.

\section{Fine-grained Nb sample treated by BCP or EP}

Nanopolished samples represent the genesis of topography from a near perfect mechanical condition; single crystal samples represent etching the grain interior surface. Here, the final set of samples represents a typical end point of polishing of SRF cavities in production [39].

\section{Characterization}

The samples were subjected to $100 \mu \mathrm{m}$ removal by $\mathrm{BCP}$ and plus $50 \mu \mathrm{m}$ removal by EP. EP done here was at $30^{\circ} \mathrm{C}$ without stirring. Samples were scanned by AFM at five locations. Representative AFM images for these conditions are shown in Fig. 15.

\section{PSD Results and analysis}

Representative PSDs for these two fine-grained $\mathrm{Nb}$ samples are shown in Fig. 16; the corresponding component fit parameters are given in Table V. 
TABLE V. Fitting parameters (unit: nm) for Fig. 16.

\begin{tabular}{|c|c|c|c|c|c|c|c|c|}
\hline & \multicolumn{3}{|c|}{$K$ correlation } & \multicolumn{2}{|c|}{ Fractal } & \multicolumn{3}{|c|}{ Shifted Gaussian } \\
\hline & $A$ & $B$ & $C$ & $K_{n}$ & $n$ & Height $\sigma$ & Size $\tau$ & Frequency $x$ \\
\hline $100 \mu \mathrm{m} \mathrm{BCP}$ & & & & $\begin{array}{r}1.1 \times 10^{-4} \\
1.5 \times 10^{-}\end{array}$ & $\begin{array}{l}3.6 \\
<10^{-3}\end{array}$ & $1.1 \times 10^{12}$ & $\begin{array}{l}2.3 \times 10^{-5} \\
10^{-5}-1.5 \times\end{array}$ & $3.3 \times 10^{-5}$ \\
\hline $\begin{array}{l}\text { Frequency range } \\
50 \mu \mathrm{m} \mathrm{EP}\end{array}$ & $6.22 \times 10^{6}$ & 1239 & 1.9 & 17.6 & 1.4 & $1.0 \times 10^{11}$ & $1.8 \times 10^{-5}$ & $2.8 \times 10^{-5}$ \\
\hline Frequency range & \multicolumn{3}{|c|}{$2 \times 10^{-4}-2.5 \times 10^{-3}$} & \multicolumn{2}{|c|}{$1 \times 10^{-3}-2 \times 10^{-4}$} & \multicolumn{3}{|c|}{$1 \times 10^{-5}-1 \times 10^{-4}$} \\
\hline
\end{tabular}

\section{Discussion}

Analysis of the PSD in terms of contributions shows that BCP-treated surfaces are dominated by the fractal or power law component, associated with the presence of sharp edges, perhaps grain boundaries. The response to EP is more complex. Significant smoothing is evident in the spatial-frequency range corresponding to $1-10 \mu \mathrm{m}$, but not longer. The dominant contributions are $K$ correlation at higher frequencies and shifted Gaussian at lower. Strikingly, a few $\mu \mathrm{m}$ removal by one process following the other results in a change to the PSD signature to that of the last-performed process.

\section{CONCLUSION}

The PSD approach affords opportunity to examine the contribution of features at different lateral scales to the observed topography. Obtaining a PSD that accurately represents the surface requires collection, preparation, and postcorrection of suitable data sets. The PSD can be further analyzed in terms of contributions related to topographic models: fractal/power law, $K$ correlation, and shifted Gaussian. Viewing the effect of BCP and EP, individually and sequentially, in these terms provides useful insights. It is particularly interesting that only a few microns removal by one process or the other causes the signature of sharp edges to come or go. The PSD approach is not, however, sensitive to the presence of a small number of prominent features, which are better revealed by height or slope histograms.

\section{ACKNOWLEDGMENTS}

This research was conducted at Thomas Jefferson National Accelerator Facility and at the College of William \& Mary. Jefferson Lab is supported by the U.S. Department of Energy under Contract No. DE-AC0506OR23177. Chen $\mathrm{Xu}$ is grateful to Jefferson Lab for support through a graduate research assistantship and personal communication with Dr. Peter Takacs.

[1] H. Padamsee, J. Knobloch, and T. Hays, $R F$ Superconductivity for Accelerators (Wiley and Sons, New York, 2008), 2nd ed.
[2] J. Knobloch, R. L. Geng, M. Liepey, and H. Padamsee, in Proceedings of the International Workshop on Radiofrequency Superconductivity (Los Alamos National Laboratory, Santa Fe, NM, 1999), p. 99.

[3] A. Dzyuba, A. Romanenko, and L. D. Cooley, Supercond. Sci. Technol. 23, 125011 (2010).

[4] Wolfgang Weingarten, CERN Report No. 19, 2011.

[5] X. Zhao, G. Ciovati, and T. R. Bieler, Phys. Rev. ST Accel. Beams 13, 124701 (2010).

[6] V. Shemelin and H. Padamsee, TESLA Technology Collaboration Report No. 2008-07, 2008.

[7] A. Gurevich, Physica (Amsterdam) 441C, 38 (2006).

[8] Hui Tian et al., Appl. Surf. Sci. 253, 1236 (2006).

[9] Hui Tian and Charles E. Reece, Phys. Rev. ST Accel. Beams 13, 083502 (2010).

[10] American Society of Mechanical Engineers standard No. B46.1 (2002).

[11] International Organization for Standardization standards No. 25178-6 (2010).

[12] Hui Tian et al., Appl. Surf. Sci. 257, 11 (2011).

[13] P. F. Chauvy, C. Madore, and D. Landolt, Surf. Coat. Technol. 110, 48 (1998).

[14] Jean Bennett and Lars Mattsson, Introduction to Surface Roughness and Scattering (Optical Society of America, Washington, DC, 1999).

[15] J. Merle Elson and Jean M. Bennett, Appl. Opt. 34, 1 (1995).

[16] William L. Briggs and Van Emden Henson, The DFT: An Owner's Manual for the Discrete Fourier Transform (Society for Industrial Mathematics, Philadelphia, PA, 1987), p. 93.

[17] E. L. Church and H.C. Berry, Wear 83, 189 (1982).

[18] E. L. Church and P.Z. Takacs, Brookhaven National Laboratory Report No. 49035, 1993.

[19] E. L. Church, T. V. Vorburger, and J. C. Wyant, Opt. Eng. 24, 3 (1985).

[20] E. L. Church and P.Z. Takacs, Brookhaven National Laboratory Report No 45578, 1991.

[21] ANSI Accredited Standards Committee Optics and Electro-Optical Standards Technical Meeting Documents Report No. 1.005, 2010.

[22] M. Senthilkumar et al., Appl. Surf. Sci. 252, 1608 (2005).

[23] Fredric J Harris, in Proceedings of the IEEE (IEEE, New York, 1978), Vol. 66, p. 1.

[24] MathWorks Matlab R2010b Documentation, http:// www.mathworks.com/help/, 2010.

[25] Hui Tian, Ph.D. thesis, College of William and Mary, 2007. 
[26] Josep Ferre Borrull, Jörg Steinert, and Angela Duparré, Surf. Interface Anal. 33, 2 (2002).

[27] Stefan Gliech, Jörg Steinert, and Angela Duparré, Appl. Opt. 41, 3224 (2002).

[28] Eugene L. Church, Appl. Opt. 27, 8 (1988).

[29] R. Jahn and H. Truckenbrodt, J. Mater. Process. Technol. 145, 40 (2004).

[30] Eugene L. Church, Peter Z. Takacs, and Thomas A Leonard, in Proceedings of SPIE, Scattering from Optical Components (SPIE, Bellingham, WA, 1989), Vol. 1165, p. 136.

[31] E.L. Church and P.Z. Takacs, Appl. Opt. 32, 19 (1993).

[32] George Palasantzas, Phys. Rev. B 48, 14472 (1993).

[33] D. Ronnow et al., Thin Solid Films 325, 92 (1998).
[34] G. Rasigni et al., Phys. Rev. B 27, 819 (1983).

[35] Angela Duparré and Stefan Jakobs, Appl. Opt. 35, 5052 (1996).

[36] Josep Ferré-Borrull, Angela Duparré, and Etienne Quesnel, Appl. Opt. 39, 31 (2000).

[37] Josep Ferré-Borrull, Angela Duparré, and Etienne Quesnel, Appl. Opt. 40, 13 (2001).

[38] D. Bloess, in Proceedings of Superconductivity Radiofrequency (CERN, Geneva, 1984), pp. 409-425.

[39] H. Tian et al., J. Electrochem. Soc. 155, 9 (2008).

[40] Xin Zhao, Sean G. Corcoran, and Michael J. Kelley, J. Appl. Electrochem. 41, 633 (2011)

[41] H. von Koch, On a Continuous Curve without Tangent Constructible, Elementary Geometry in Classics on Fractals (Westview Press, Boulder, CO, 2004), p. 2545. 